This item was submitted to Loughborough's Research Repository by the author.

Items in Figshare are protected by copyright, with all rights reserved, unless otherwise indicated.

\title{
Rapid assessment of site specific DNA methylation through resistive pulse sensing
}

PLEASE CITE THE PUBLISHED VERSION

https://doi.org/10.1021/acssensors.7b00935

PUBLISHER

(C) American Chemical Society

VERSION

AM (Accepted Manuscript)

\section{PUBLISHER STATEMENT}

This work is made available according to the conditions of the Creative Commons Attribution-NonCommercialNoDerivatives 4.0 International (CC BY-NC-ND 4.0) licence. Full details of this licence are available at: https://creativecommons.org/licenses/by-nc-nd/4.0/

\section{LICENCE}

CC BY-NC-ND 4.0

\section{REPOSITORY RECORD}

Healey, Matthew, William Rowe, Sofia Siati, Muttuswamy Sivakumaran, and Mark Platt. 2018. "Rapid Assessment of Site Specific DNA Methylation Through Resistive Pulse Sensing”. Loughborough University. https://hdl.handle.net/2134/32424. 


\title{
Rapid Assessment of Site Specific DNA Methylation through Resistive Pulse Sensing
}

Matthew J. Healey, ${ }^{a}$, William Rowe, ${ }^{a}$, Sofia Siati ${ }^{\mathrm{a}}$, Muttuswamy Sivakumaran, ${ }^{\mathrm{b}}$ and Mark Platt ${ }^{\mathrm{a}, *}$

\footnotetext{
${ }^{a}$ Department of Chemistry, Loughborough University, Loughborough, Leicestershire, LE11 3TU, United Kingdom

${ }^{\mathrm{b}}$ Peterborough City Hospital, Edith Cavell Campus, Bretton Gate, Peterborough, PE3 9GZ, United Kingdom
}

* Corresponding author: M. Platt: m.platt@lboro.ac.uk, +44(0)1509222573

\begin{abstract}
Many diseases are defined by patterns of DNA methylation which result in aberrant gene expression. We present a rapid assay based upon Resistive Pulse Sensing, RPS, to characterise sequence specific DNA methylation sites in genomic DNA. We modify the surface of superparamagnetic beads, SPBs, with DNA (capture probe). The particles are added to solution where they bind to and extract sequence specific DNA (Target DNA). The target loaded SPBs are then incubated with antibodies which bind to the methylation sites, and the velocity of the SPBs through the nanopore reveals the number and location of the epigenetic markers within the target. The approach is capable of distinguishing between different methylation sites within a DNA promoter region. Crucially the approach is not dependant on accurate sequencing of assayed DNA, with genomic regions targeted through complimentary probes. As such the number of stages and reagents costs are low and the assay is complete in under 60 mins which includes the incubation and run times. The format also allows simultaneous quantification of number of copies of methylated DNA, and we illustrate this with a dose response curve.
\end{abstract}

Keywords: nanopore, resistive pulse sensor, epigenetics, methylation, bioassay 
One of the most challenging goals in modern bioscience remains; how can we understand disease through analysis of biological variation? In the post genomic era, quantitative bioscience has been dominated by seeking the answer through analysis of genomic and transcriptomic variation. This arises not from an ad-hoc understanding of the pre-eminence of these components, but rather the relative ease, scope and reliability of the underlying measurements. As our repertoire of quantitative tools expands and improves, so does our understanding of the role of metabolomic ${ }^{1}$, proteomic ${ }^{2}$ and epigenetic ${ }^{3}$ dysregulation in disease.

The role of DNA methylation has been shown to be fundamental to many processes including aging ${ }^{4}$, exercise and cancer $^{3}$. In fact cancer specific DNA methylation patterns have been observed across many tumours ${ }^{5}$. Despite the potential utility of methylation assays their adoption in the clinic and the lab has been far from absolute due to inherent challenges. Methods for measuring DNA methylation often rely on chemical modification through bisulfite treatment ${ }^{6}$. Treatment of cytosine with bisulfite leads to conversion to uracil, a reaction that is prevented in 5-methylcytosine. The modified base (or lack thereof) can be identified through sequencing or hybridisation of sequence specific primers and subsequent PCR amplification. Many problems arise with bisulfite conversion and subsequent assay, including DNA degradation, reaction by-products leading to alternative modifications, a requisite for large sample quantities, PCR bias, etc ${ }^{7}$.

Recent studies have demonstrated the potential of solid state nanopores in identifying DNA methylation sites ${ }^{8-10}$. By binding MBD (methyl binding domain) proteins to 5-methylcytosine sites, they were able to observe a 3-fold increase ionic blockage current relative to unmethylated DNA. The technique avoids the need for bisulfite conversion and its inherent problems, yet requires detailed sequence analysis on the nanopore platform.

Tunable Resistive Pulse Sensing (TRPS) is a solid state nanopore technique based upon the Coulter principle ${ }^{11,12}$. Such technologies have been applied to the characterisation of biological and nanomaterials ${ }^{11,13-17}$. We have previously demonstrated the capacity of the technique to quantify DNA protein interactions, in the form of a bound protein biomarker to a DNA aptamer ${ }^{18-21}$. In the assay DNA was conjugated to superparamagnetic beads, SPBs, creating a negatively charged surface. Protein binding to this DNA changes the charge density around the particle and consequently reduces its velocity as it traverses the nanopore. The decrease is proportional to the concentration of protein in solution.

Rassf1a is a tumour suppressor gene, repression of which has been associated with a range of cancers ${ }^{22}$. Hypermethylation of CpG islands in the promoter of Rassf1a is prognostic of its dysregulation, with degree of methylation inversely correlated with gene expression ${ }^{23}$. We assess the ability of TRPS to determine the level of methylation in a proportion (54 bases) of the promoter region of Rassf1a. Anti-5methylcytosine antibody (an antibody that specifically binds methylation sites in 
single stranded DNA) is bound to the promoter (target), which is anchored to a nanoparticle through a complementary (capture) probe. Measuring the degree to which the DNA charge is shielded by the antibody will determine the level of methylation. Crucially the assay negates the requirement of the pore to sequence the DNA, with specific genomic regions targeted through hybridisaton to complementary probes.

The aim is to produce a rapid, reliable assay for methylation, with applications ranging from the clinic to the farming industry ${ }^{24}$. We find the assay is highly sensitive in discriminating between methylated and unmethylated sequences. In addition methylation sites at different points in the sequence could be distinguished in the ion blockage profiles of the nanoparticles as they traversed the pore. The assay also correlates particle velocity with number of methylations sites in the promoter region, where by the presence of single or double epigenetic markers can be identified. The number of methylated target DNAs in solution allows a dose response curve for the quantification of methylated DNA in solution over two orders of magnitude.

\section{Experimental}

\section{Chemicals and Reagents}

A 54 base region from the Rasf1a promoter sequence from chromosome III of the human genome was selected (5'-GG[met1-C]CCGCCCTGTGGCCCCG[met2C]CCGGCCCGCGCTTGCTAGCGCCCAAAGCCAGCGA-3'), this is to simulate the product of a restriction enzyme digest. A 30 base capture probe complementary to the the Rassf1 promotor sequence, was purchased from Sigma Aldrich Chemicals with a 5' biotin tag and 5T linker (biotinTTTTTTCGCTGGCTTTGGGCGCTAGCAAGC). In addition four variations of the Rassf1a promoter: unmethylated ControlDNA, 5' single methylation (at site met1-C above EndDNA), 3' single methylation (site met2-C MiddleDNA) and double methylation (sites met1-C and met2-C DoubleDNA), were also purchased in lyophilised powders with RP1 purification from Sigma Aldrich Chemicals (UK). Anti5-methylcytosine antibody at $1 \mathrm{mg} / \mathrm{mL}$ (ab73938) was purchased from Abcam, UK. The concentration of the antibody was verified using UV-Vis spectroscopy (Nanodrop 2000, ThermoFisher Scientific). Using the average MW of the antibody $(970000 \mathrm{~g} / \mathrm{Mol})$ the number of antibodies per unit volume could be established and used for ratio calculations. Water purified to a resistivity of $18.2 \mathrm{M} \Omega \mathrm{cm}$ (Maxima) was used to make all solutions. Phosphate buffered saline (PBS, P4417 (1× PBS tablet $(0.01 \mathrm{M}$ phosphate buffer, $0.0027 \mathrm{M}$ potassium chloride, $0.137 \mathrm{M}$ sodium chloride) in $200 \mathrm{~mL}$ deionised water $(18.2 \mathrm{M} \Omega \mathrm{cm})$ ). ) was purchased from Sigma Aldrich (UK) and used unmodified through the experiment. Carboxylated polystyrene beads, denoted as CPC200, with a mean nominal diameter of $210 \mathrm{~nm}$ and stock concentration of $1 \times 10^{12}$ particles $/ \mathrm{mL}$, were purchased from Bangs Laboratories, USA and used as a concentration calibrant at $2 \times 10 \% / \mathrm{mL}$. Prior to use the beads were vortexed for $30 \mathrm{~s}$ and sonicated for $1 \mathrm{~min}$ to ensure monodispersity. 
$120 \mathrm{~nm}$ streptavidin coated beads (Ademtech 03211) were used and functionalized with the biotin labelled capture probe with the Rassf1a promoter sequence as follows: Equal volumes of oligonucleotides, corresponding to $100 \%$ of the beads binding capacity (calculated using the manufacturer's specification) of the streptavidin modified beads, were vortexed for $30 \mathrm{~s}$ prior to heating to $95^{\circ} \mathrm{C}$ for 5 min in a mini drybath (Benchmark Scientific, USA). The reaction mixture was vortexed again for $30 \mathrm{~s}$ and allowed to cool to RT for $30 \mathrm{~min}$ on a rotary wheel before the addition of beads. The mixture was placed on a MagRack (Life Science) for 10 min until a cluster of beads was visible. The solution was carefully removed and replaced with an equal volume of PBS. The concentration of beads was kept constant throughout all experiments at $2 \times 10^{9} / \mathrm{mL}$.

\section{Concentration Analysis}

Anti-5-methylcytosine antibody (a pentameric IgM) was added to the DNA hybridised beads. The ratio of antibodies to beads varied from 0 up to three orders of magnitude of antibodies per bead. After the addition of the antibody the mixtures were vortexed for $30 \mathrm{~s}$ and placed on a rotary wheel for $8 \mathrm{~min}$ and prior to TRPS analysis the mixture was vortexed for $15 \mathrm{~s}$ and sonicated for $5 \mathrm{~s}$.

\section{Dose Response}

Full-Binding Capacity Experiment - The concentration of Rassf1 was maintained at $100 \%$ relative to the binding capacity of the beads, to which varied amounts of complementary DNA equivalent to $0,10,20,40,60,80$, and $100 \%$ of the bead's binding capacity were added. The bound DNA sequences were then hybridised to the beads as previously described. The concentration of anti-5-methylcytosine antibody was maintained at three orders of magnitude relative to the bead concentration.

Half-Binding Capacity Experiment - Throughout this set of experiments the Rassf1 concentration was kept constant $-50 \%$ of the beads binding capacity. The bead binding capacity is calculated to be half using the manufacturer's specifications and assumed from previous studies ${ }^{21,25}$. The amount of complementary DNA varied from $0,10,20,30$, and $40 \%$ of the beads binding capacity. As previously described the bound DNA was hybridised to the beads with the concentration of anti-5methylcytosine antibody maintained at three orders of magnitude relative to the bead concentration.

TRPS Analysis

TRPS was performed using the qNano system purchased from IZON Science (New Zealand). The technique uses elastomeric tunable nanopores with Izon's own data capturing software Izon Control Suite (V3.1.2.53). NP200 pores were used, suitable 
for analysing beads between 85 and $500 \mathrm{~nm}$ (stated by the manufacturer). An appropriate stretch and voltage were applied throughout so that the blockades of CPC200s in PBS were at least $0.5 \mathrm{nA}$ above the background noise; a measure to account for the variations in pore manufacturing. The operation of the qNano is described elsewhere ${ }^{12,18,26}$ but briefly: the lower fluid cell was filled with $75 \mu \mathrm{L}$ of PBS, ensuring no air bubbles are present and the upper fluid cell contained $40 \mu \mathrm{L}$ of the sample. After each measurement the sample was removed from the upper fluid cell and replaced with PBS. This was repeated several times, applying varying amounts of pressure and vacuum, until visible blockades were no longer observed. Samples were replicated in triplicate, unless stated with $\mathrm{H} 15$ estimates of the mean reported and used for normalisation.

\section{Calculating the particle velocities}

The method used the resistive pulse to calculate the relative velocity, and identifies the point of greatest resistance in the signal trace (the resistive blockade peak). For each blockade, the time at which the peak occurs is defined as $\mathrm{T}_{1.0}$ (time at $100 \%$ of peak magnitude) and the maximum magnitude of the pulse (relative to the local baseline resistance) is recorded as dRmax. Here the value at $T_{0.5}$ (width of the pulse at $50 \%$ of the peak magnitude is used $)^{25,27}$. To keep the method simple and applicable in further applications the actual zeta potential of the particle is not calculated and for further simplicity in the subsequent figures we use only one measurement to represent the particle speed which is $1 / T_{0.50}$.

\section{Results and Discussion}

The outline for the assay is shown in fig 1. Here a synthesised target is used, however it should be noted the concept can be adapted to any working assay where the genomic DNA is first extracted and digested prior to analysis. A biotinylated capture probe is added to the DNA sample (fig1A). The capture probe was designed to be a perfect complement to a flanking region of the Rassf1a gene where the methylation is known to occur. The mixture was heated to $95^{\circ} \mathrm{C}$ for $5 \mathrm{~min}$ to denature the target DNA. The mixture was then allowed to cool to RT for 30 min allowing the biotinylated probe to hybridise to its target. Streptavidin coated superparamagetic beads, SPBs, were then added to the solution, mixed for 5 min (fig1B) and extracted using a magnetic rack before being finally placed into PBS buffer. During this extraction the aim was to allow the SPBs to bind the biotinytated capture DNA along with the hybridised target DNA (fig 1D). 


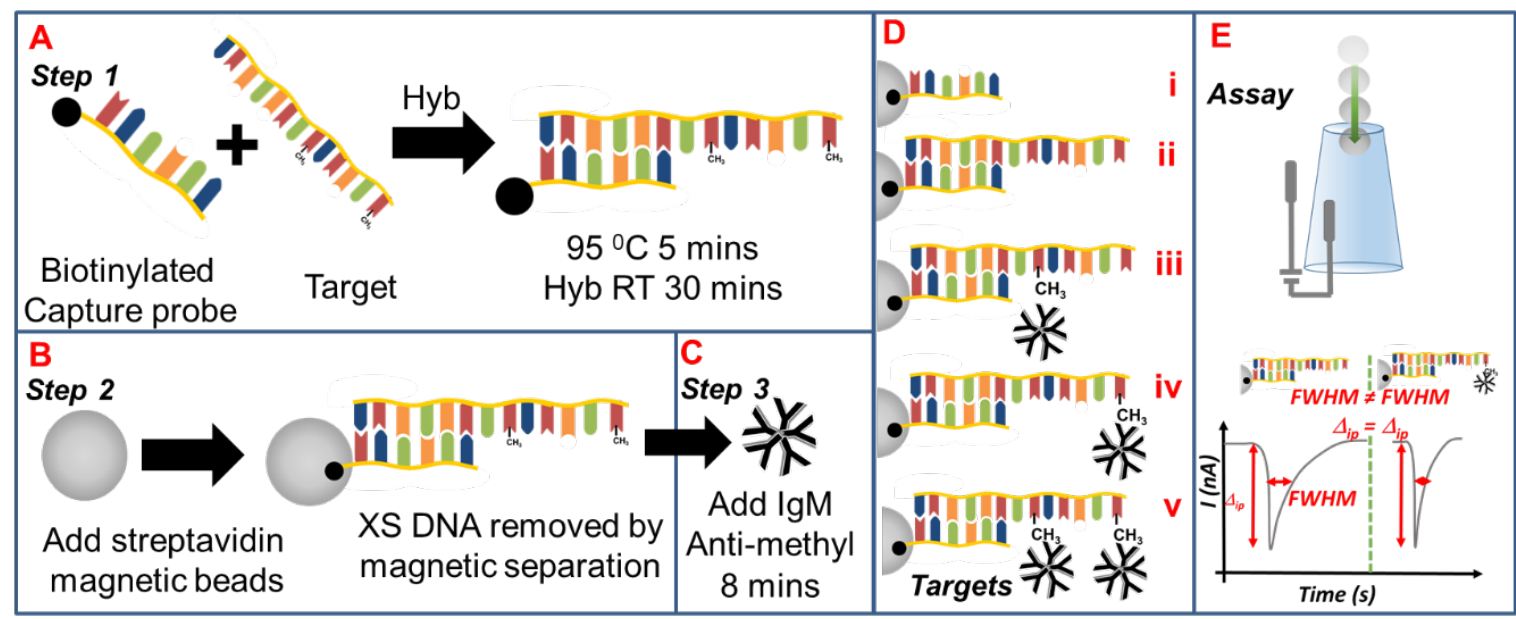

Fig 1. Schematic of the assay. A) Biotinyated Capture probe is incubated with the target DNA. B) Streptavidin coated SPBs capture the DNA. C) Antibody is added to the solution. D) Depending upon the target DNA present the SPBs have $i$ - Capture DNA, ii - unmethylated Target DNA, iii - single methylated site (MidDNA) iv - single methylation (EndDNA) or $v$ - two methylation sites (DoubleDNA). $E-T h e$ velocity of the particles through the RPS reveals which target is present.

The concentration of SPBs added to the solution was adjusted such that their total binding capacity was equal to the concentration of biotinylated DNA. This results in the extracted SPBs always having a coating of DNA even in the absence of target DNA (Fig1Di). We have recently shown how the length and concentration of single and double stranded DNA on SPBs can be monitored using TRPS ${ }^{26}$. We utilise the same concept and signal transduction mechanism here. In summary the binding of DNA to the SPBs results in the zeta potential of the particles becoming more negative. The change in zeta potential is measured by monitoring the translocation velocity of the particles through the pore, and measured via the full width half maximum, FWHM, (fig1E). Increasing the number of DNA molecules or the length of the sequence around each SPB results in an increase in zeta potential, inferred by an increase in $1 / T_{0.5}$ i.e. more negatively charged particles move through the pore quicker. An example of this is shown in fig 2. The relative velocity of the particles is then plotted as the reciprocal of $\mathrm{T}_{0.5}$ and shown for Streptavidin SPBs (blank), capture probe modified SPBs (sSDNA) and target probe modified SPBs (dsDNA). The relative order of the velocity of the three particles shows that the target DNA is faster than capture probe only, and the slowest particles are the blank SPBs. 

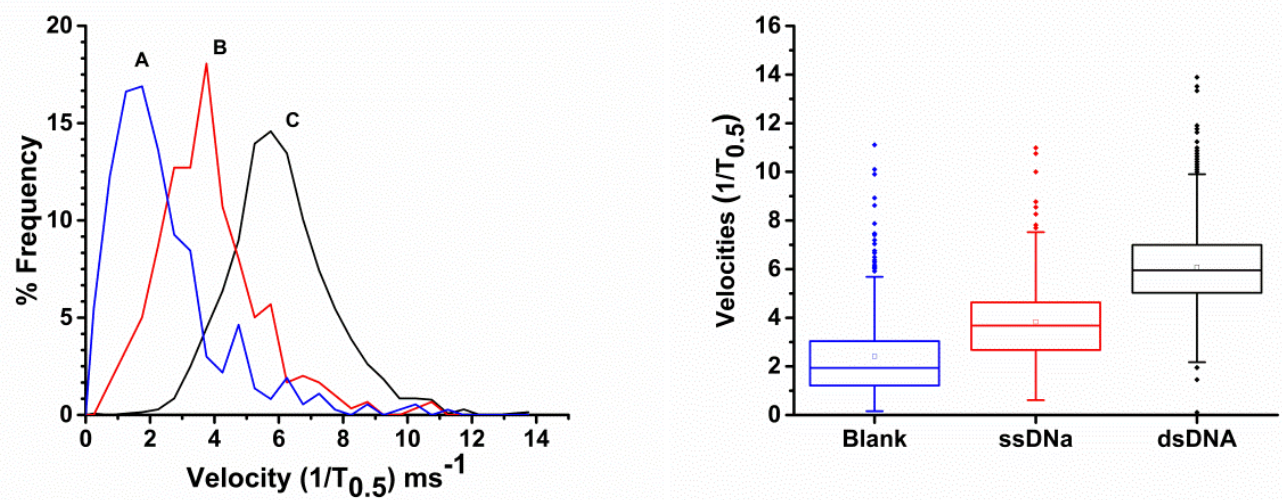

Fig 2. Left, Plot of $1 / T_{0.5}$ versus frequency for SPB modified with no DNA curve $A$ (Blank). Capture probed modified DNA (sSDNA) curve $B$, and SPBs with target DNA curve C (dsDNA). Right, same data visualised as box-whisker plots. Events for DNA type and blank beads $>500$

Here we do not convert the translocation velocities into zeta potential values, as it's not required and adds an extra stage within the calibration. Monitoring the relative change in velocities of the particles is sufficient to determine the presence of the DNA.

Having captured the target DNA, the next stage was to assess if the methylated bases within the target DNA could be identified. Two samples were prepared, the first was a target DNA with no methylation makers, and the second with a single methylation point approx. half way within the sequence (MidDNA) fig 1D ii and iii, respectively. The anti-methyl antibody was incubated with the SPBs and if epigenetic markers are present, they should bind to the DNA. We have recently demonstrated how the TRPS system can monitor Protein-DNA interactions and is the basis for many aptamer based sensors. The binding of the antibody to the DNA changes the charge density around the particles and results in a change in particle velocities $^{18,20,21,28}$. A decrease in velocity (with respect to a positive bias below the pore) is thought to be due to several parameters. Changes to the DNA structure as it binds the protein may require an increased number of counter ions to stabilise any tertiary structure, secondly the protein's $\mathrm{pl}$ is between 6-7.4, and therefore has a net (and slight) positive charge for the $\mathrm{pH}$ used in the experiment, which can counter the charge on the DNA backbone. In addition, the binding of the protein (even if in a net neutral charge state) to the DNA will disrupt the double layer structure and affect the electrophoretic velocity.

To test this hypothesis and to optimise the amount of antibody required, we titrated differing Bead:Antibody ratios shown in fig 3a (and Fig S1). For the methylated sample, as the number of antibodies per bead increases from 0 up to three orders of magnitude ( $1: 1000)$, the particle velocity decreases as they bind to the DNA. Whilst there is a selective interaction between the antibody and methylation site, there will be some nonspecific interactions between the antibody and DNA. Thus at the larger concentrations of antibody we attribute the change in velocity for the nonmethylated DNA to nonspecific interactions between the IgM and the DNA/ beads surface. It 
should also be noted that whilst the binding of the antibody changes the relative velocity of the particle, we do not observe within the current setup a change in particle volume.

We repeated this experiment using target DNA which contained a single methylation site at a different location further from the bead surface (EndDNA) Fig 1Div, and a target DNA which contained two methylation sites (DoubleDNA) Fig 1Dv. Similar trends were observed i.e. as the antibody number increased the particle velocity decreased at a rate much higher than the control. It should be noted that in no case did we see particle aggregation, and the particle size distribution plots are given in supplementary fig S2. In the presence of nonspecific interactions taking place at higher antibody numbers it was concluded that a ratio of three orders of magnitude more antibodies to bead was a suitable ratio to proceed with further experiments.

A

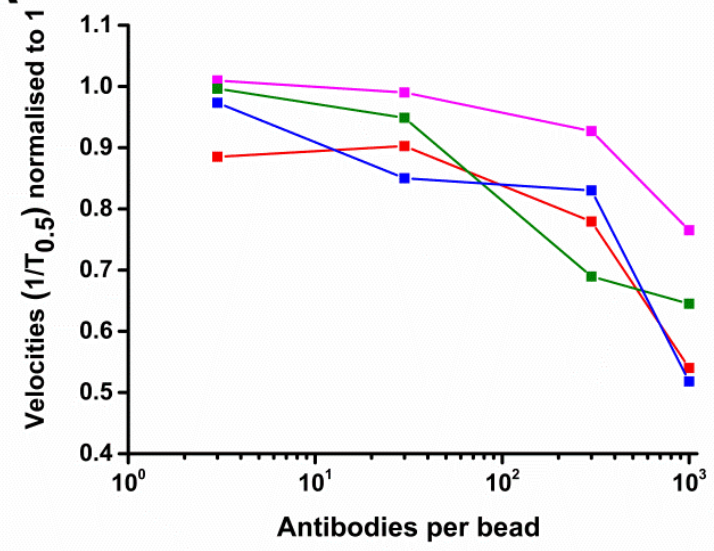

B

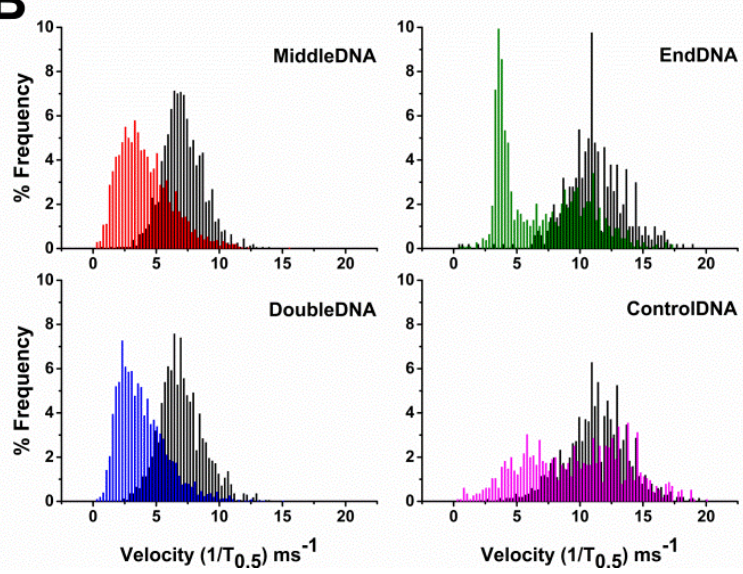

Fig 3 (a) Normalised $1 / T_{0.5}$ values as a function of antibody per SPB. The ratio of SPBs:antibody was varied for all four types DNA used to determine methylation site. Magenta shows data for ControlDNA, in which no methylation is present; green represents EndDNA; red MiddleDNA; and blue the doubly methylated DoubleDNA (b) Translocation velocities distributions taken from $1 / T_{0.5}$ of the four $D N A$ types. The ratio of SPBs:Antibody is constant at three orders of magnitude more antibodies per SPBs, with the concentration of capture DNA and target equivalent to 100\% of the SPBs binding capacity. Black distributions represent when no antibody is present. Events for each data point $>400$.

We hypothesised that the location at which the antibody bound to the DNA, i.e. if the methylation site was in the Mid, End or Double, may result in different magnitudes of velocity change helping identify the location of the methylation site. However as can be seen in fig3B the mean velocity change and distribution of velocities for hundreds of particles are similar in this design of the experiment.

From the above experimental design, the presence of the epigenetic marker could be easily identified. To quantify the number of DNA copies in solution which contained these markers, we prepared a dose response curve. To demonstrate this we chose the MidDNA. In this experiment the number of SPBs, the concentration of capture DNA and antibody ratio was kept constant. The concentration of target DNA was varied and the results shown in fig $4 \mathrm{a}$. The dose response curve shows a change over 2 orders of magnitude. As the number of target DNA increases the 
velocity of the particles decrease. At lower concentrations of target DNA most of the SPBs surface is covered with biotinylated capture probe, and as it does not bind to the antibody, its negative charge dominates the particles velocity. To improve the sensitivity of the dose response curve at lower concentrations of target DNA, we lowered the number of capture probes to be circa $50 \%$ of the binding capacity of the beads, i.e. keeping every other parameter the same as fig 4 a but the concentration of the capture probe was halved. The ratio of the bead concentration, binding capacity, capture probe capacity and numbers of antibodies are given in table 1.

\begin{tabular}{ccc}
\hline Bead to antibody ratio* & $\begin{array}{c}\text { Capture Probe } \\
\text { Concentration Relative to } \\
\text { the Bead's Binding } \\
\text { Capacity (\%) }\end{array}$ & $\begin{array}{c}\text { Target EndDNA } \\
\text { Concentration } \\
\text { Relative to the Bead's } \\
\text { Binding Capacity (\%) }\end{array}$ \\
\hline $1: 1000$ & 100 & 0 \\
$1: 1000$ & 100 & 10 \\
$1: 1000$ & 100 & 20 \\
$1: 1000$ & 100 & 40 \\
$1: 1000$ & 100 & 60 \\
$1: 1000$ & 100 & 80 \\
$1: 1000$ & 100 & 100 \\
$1: 1000$ & & 0 \\
$1: 1000$ & 50 & 10 \\
$1: 1000$ & 50 & 20 \\
$1: 1000$ & 50 & 30 \\
$1: 1000$ & 50 & 40 \\
\hline
\end{tabular}

Table 1. Concentration of SPB, capture probe and antibodies in Assays. *the bead to antibody ratio is an approximation and is referred to as three orders of magnitude in the text

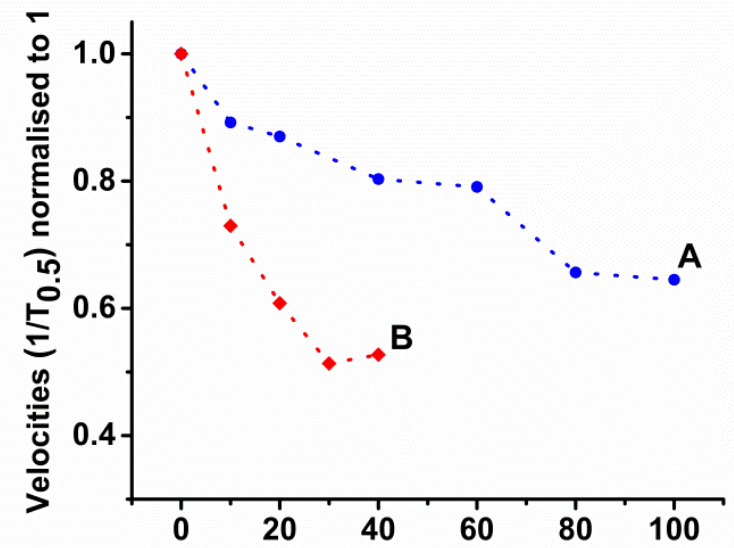

$\%$ of EndDNA relative to the bead's binding capacity

Fig 4 Concentration of beads was kept constant at $2 \times 10^{9} / \mathrm{mL}$. (a) Concentration of capture probe is $100 \%$ relative to the binding capacity of the bead. The concentration of target EndDNA was varied from $0-100 \%$, relative to the binding capacity of the bead. (b) Concentration of capture probe is 50\%, relative to the binding capacity of bead. Again the concentration of target EndDNA was varied from 0 $-40 \%$ of the beads binding capacity. Events for each data point $>500$ 
As can be seen in fig 4B halving the capture probe concentration resulted in a sharp decrease in velocity of the SPBs and the concentration of target DNA was increased from $0 \mathrm{nM}$ to $119 \mathrm{nM}$. The dynamic range of this assay is lower than that in fig $4 \mathrm{~A}$ as the beads can only capture half the concentration of target DNA, and at concentrations above $47.6 \mathrm{nM}$, there was no further decrease in velocity. Owing to the rapid change in velocity for the lower concentrations of capture probe we again measured the $1 / T_{0.5}$ for the Mid, End and DoubleDNA targets to see if their relative velocities differentiated. Table 2 shows the change in velocity for the same concentrations of SPB, antibody and target DNA for the different variations of epigenetic target DNA.

\begin{tabular}{cccc}
\hline DNA Type & $\begin{array}{c}\text { Mean Velocity, 1/T } 0.5 \\
\mathrm{~ms}\end{array}$ & Standard Deviation & Velocity Decrease, \% \\
\hline Double & 2.76 & 0.36 & 50.73 \\
Middle & 3.39 & 0.31 & 39.48 \\
End & 2.94 & 0.41 & 47.56 \\
Blank & 5.60 & 0.22 & - \\
\hline
\end{tabular}

Table 2. Relative velocity for the SPB modify with capture DNA (Blank), Mid, End and Double DNA. The concentration of DNA was 50\% relative to the binding capacity of the beads, 30\% target DNA, beads $2 \times 10^{9} / \mathrm{mL}$ and antibody three orders of magnitude more antibody per bead. Events for each DNA type, and blank >600 (blank 2303; MiddleDNA 932; EndDNA 979; and DoubleDNA 686).

Table 2 shows that the largest change in velocity was observed for the double methyl target, followed by the end and middle. At the lower concentrations of capture probe the relative velocity of the beads allows differentiation of the location of the epigenetic marker. At these lower concentrations of capture probe the average distance between neighbouring DNA on the SPB's surface increases, this may allow the antibody-DNA to adopt a different structure/ bind easier than in the fully packed SPB experiment. Although it should be noted that the middle methylation target still shows a change in velocity and thus binding to the antibody must still take place.

\section{Conclusion}

We demonstrate the characterisation of methylated DNA sequences through TRPS. The process allows for the rapid quantification of epigenetic markers with insight into the location of the marker within the target. The capacity of the technique to quickly and reliably quantify nucleic acid-protein interactions presents a platform for the potential analysis of: polysome gradients (translation rate), RNA methylation (anti-5methylcytosine also binds methylated RNA), protein phosphorylation and histone acetylation. This is in addition to previous studies that have demonstrated the ability of TRPS to quantify SNPS, microvesicles, protein biomarkers etc. TRPS is a burgeoning technology that lends itself to multiomic analysis, we highlight its application in the characterisation of methylation sites within DNA. Further optimisation of the approach may yield a multipurpose tool for the clinic capable of analysing biological variation beyond the scope of current technologies. 


\section{Associated Content}

Supporting Information Available: The following files are available free of charge. Blockade magnitude distributions for the assays and concentration analysis graphs.

\section{Conflicts of interest}

All authors declare no conflicts of interest.

\section{References}

(1) Gowda, G. A. N.; Zhang, S.; Gu, H.; Asiago, V.; Shanaiah, N.; Raftery, D. Metabolomics-Based Methods for Early Disease Diagnostics. Expert Rev. Mol. Diagn. 2008, 8 (5), 617-633.

(2) Hanash, S. Disease Proteomics. Nature 2003, 422, 226.

(3) Portela, A.; Esteller, M. Epigenetic Modifications and Human Disease. Nat. Biotechnol. 2010, 28, 1057.

(4) Horvath, S. DNA Methylation Age of Human Tissues and Cell Types. Genome Biol. 2013, 14 (10), 3156.

(5) Witte, T.; Plass, C.; Gerhauser, C. Pan-Cancer Patterns of DNA Methylation. Genome Med. 2014, 6 (8), 66.

(6) Clark, S. J.; Harrison, J.; Paul, C. L.; Frommer, M. High Sensitivity Mapping of Methylated Cytosines. Nucleic Acids Res. 1994, 22 (15), 2990-2997.

(7) Genereux, D. P.; Johnson, W. C.; Burden, A. F.; Stöger, R.; Laird, C. D. Errors in the Bisulfite Conversion of DNA: Modulating Inappropriate- and FailedConversion Frequencies. Nucleic Acids Res. 2008, 36 (22), e150-e150.

(8) Shim, J.; Kim, Y.; Humphreys, G. I.; Nardulli, A. M.; Kosari, F.; Vasmatzis, G.; Taylor, W. R.; Ahlquist, D. A.; Myong, S.; Bashir, R. Nanopore-Based Assay for Detection of Methylation in Double-Stranded DNA Fragments. ACS Nano 2015, 9 (1), 290-300.

(9) Rand, A. C.; Jain, M.; Eizenga, J. M.; Musselman-Brown, A.; Olsen, H. E.; Akeson, M.; Paten, B. Mapping DNA Methylation with High-Throughput Nanopore Sequencing. Nat. Methods 2017, 14, 411.

(10) Lepoitevin, M.; Ma, T.; Bechelany, M.; Janot, J.-M.; Balme, S. Functionalization of Single Solid State Nanopores to Mimic Biological Ion Channels: A Review. Adv. Colloid Interface Sci. 2017, 250, 195-213.

(11) Kozak, D.; Anderson, W.; Vogel, R.; Chen, S.; Antaw, F.; Trau, M. Simultaneous Size and $\zeta$-Potential Measurements of Individual Nanoparticles in Dispersion Using Size-Tunable Pore Sensors. ACS Nano 2012, 6 (8), 69906997.

(12) Blundell, E. L. C. J.; Mayne, L. J.; Billinge, E. R.; Platt, M. Emergence of Tunable Resistive Pulse Sensing as a Biosensor. Anal. Methods 2015. 
(13) Qiu, Y.; Siwy, Z. Probing Charges on Solid-Liquid Interfaces with the ResistivePulse Technique. Nanoscale 2017, 9 (36), 13527-13537.

(14) Buchsbaum, S. F.; Nguyen, G.; Howorka, S.; Siwy, Z. S. DNA-Modified Polymer Pores Allow pH- and Voltage-Gated Control of Channel Flux. J. Am. Chem. Soc. 2014, 136 (28), 9902-9905.

(15) Harrell, C. C.; Kohli, P.; Siwy, Z.; Martin, C. R. DNA-Nanotube Artificial Ion Channels. J. Am. Chem. Soc. 2004, 126 (48), 15646-15647.

(16) Lan, W.-J.; Kubeil, C.; Xiong, J.-W.; Bund, A.; White, H. S. Effect of Surface Charge on the Resistive Pulse Waveshape during Particle Translocation through Glass Nanopores. J. Phys. Chem. C 2014, 118 (5), 2726-2734.

(17) Weatherall, E.; Hauer, P.; Vogel, R.; Willmott, G. R. Pulse Size Distributions in Tunable Resistive Pulse Sensing. Anal. Chem. 2016, 8648-8656.

(18) Billinge, E. R.; Platt, M. Multiplexed, Label-Free Detection of Biomarkers Using Aptamers and Tunable Resistive Pulse Sensing (AptaTRPS). Biosens.

Bioelectron. 2015, 68, 741-748.

(19) Billinge, E. R.; Platt, M. Aptamer Based Dispersion Assay Using Tunable Resistive Pulse Sensing (TRPS). Anal. Methods 2015, 7 (20), 8534-8538.

(20) Billinge, E. R.; Broom, M.; Platt, M. Monitoring Aptamer-Protein Interactions Using Tunable Resistive Pulse Sensing. Anal. Chem. 2013, 86 (2), 1030-1037.

(21) Blundell, E. L. C. J.; Mayne, L. J.; Christie, S. D. R.; Platt, M. Protein Detection Using Tunable Pores: Resistive Pulses and Current Rectification. Faraday Discuss. 2016, No. 193, 487-505.

(22) Burbee DG1, Forgacs E, Zöchbauer-Müller S, Shivakumar L, Fong K, Gao B, Randle D, Kondo M, Virmani A, Bader S, Sekido Y, Latif F, Milchgrub S, Toyooka S, Gazdar AF, Lerman MI, Zabarovsky E, White M, Minna JD. Epigenetic Inactivation of RASSF1A in Lung and Breast Cancers and Malignant Phenotype Suppression. J. Natl. Cancer Inst. 2001, 93 (9), 691-699.

(23) Kajabova, V.; Smolkova, B.; Zmetakova, I.; Sebova, K.; Krivulcik, T.; Bella, V.; Kajo, K.; Machalekova, K.; Fridrichova, I. RASSF1A Promoter Methylation Levels Positively Correlate with Estrogen Receptor Expression in Breast Cancer Patients. Transl. Oncol. 2013, 6 (3), 297-304.

(24) Ong-Abdullah, M.; Ordway, J. M.; Jiang, N.; Ooi, S-E.; Kok, S-Y.; Sarpan, N.; Azimi, N.; Hashim, A. T.; Ishak, Z.; Rosli, S. K.; Malike, F. A.; Bakar, N. A. A.; Marjuni, M.; Abdullah, N.; Yaakub, Z.; Amiruddin, M. D.; Nookiah, R.; Singh, R.; Low, E-T. L.; Chan, K-L.; Azizi, N.; Smith, S. W.; Bacher, B.; Budiman, M. A.; Van Brunt, A.; Wischmeyer, C.; Beil, M.; Hogan, M.; Lakey, N.; Lim, C-C.; Arulandoo, X.; Wong, C-K.; Choo, C-N.; Wong, W-C.; Kwan, Y-Y.; Alwee, S. S. R. S.; Sambanthamurthi, R.; Martienssen, R. A. Loss of Karma Transposon Methylation Underlies the Mantled Somaclonal Variant of Oil Palm. Nature 2015, 525, 533.

(25) Blundell, E. L. C. J.; Vogel, R.; Platt, M. Particle-by-Particle Charge Analysis of 
DNA-Modified Nanoparticles Using Tunable Resistive Pulse Sensing. Langmuir 2016, 32 (4), 1082-1090.

(26) Blundell, E. L. C. J.; Vogel, R.; Platt, M. Particle-by-Particle Charge Analysis of DNA-Modi Fi Ed Nanoparticles Using Tunable Resistive Pulse Sensing. 2015.

(27) Mayne, L. J.; Christie, S. D. R.; Platt, M. A Tunable Nanopore Sensor for the Detection of Metal Ions Using Translocation Velocity and Biphasic Pulses. Nanoscale 2016, 8 (45), 19139-19147.

(28) Platt, M.; Willmott, G. R.; Lee, G. U. Resistive Pulse Sensing of AnalyteInduced Multicomponent Rod Aggregation Using Tunable Pores. Small 2012, 8 (15), 2436-2444. 
For TOC only

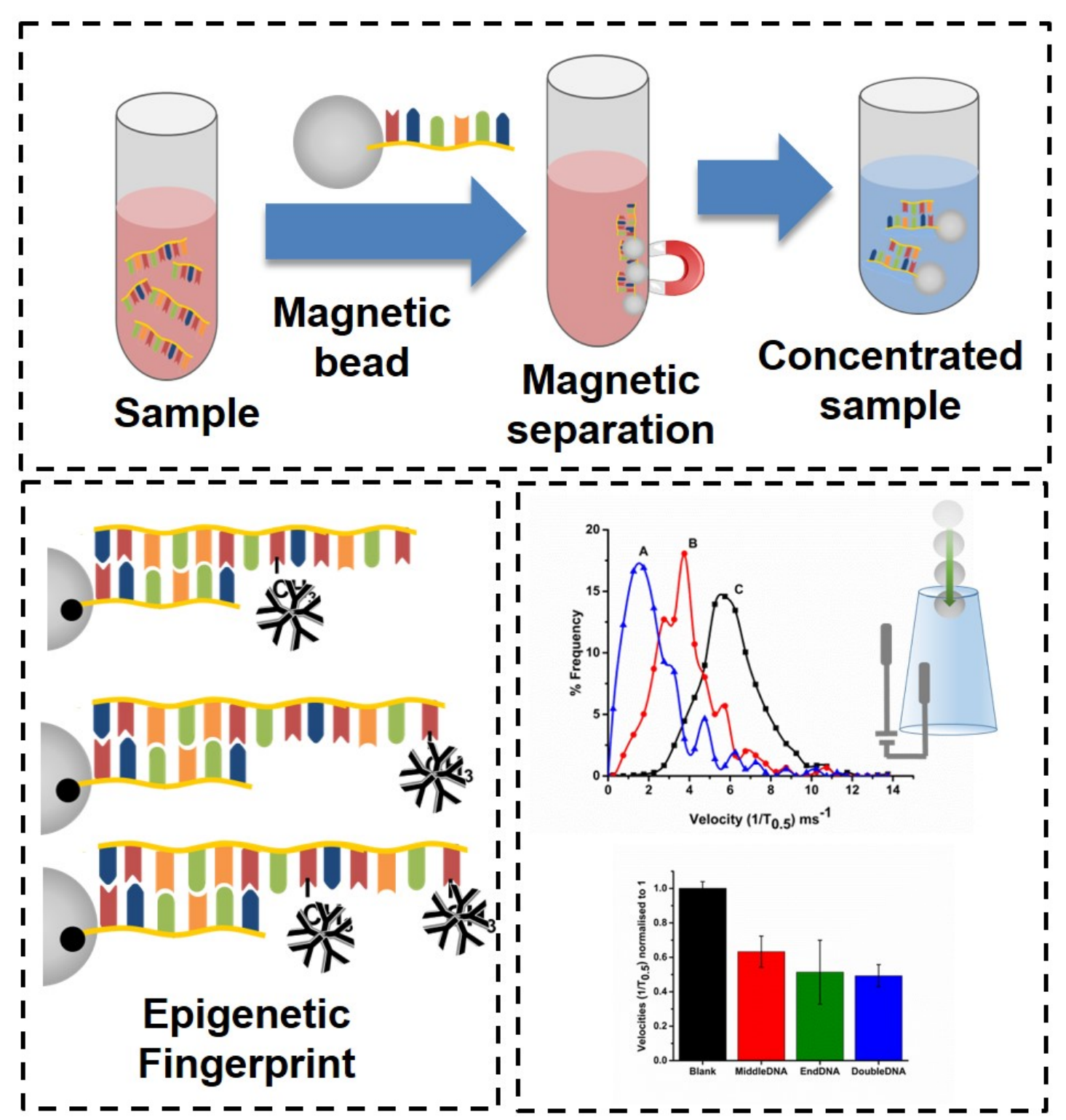

Superparamagnetic particles extract sequence specific DNA. The binding of antibodies to the target reveals the number and position of the epigenetic markers, as well as the number of copies of DNA in solution. 